\title{
Microstructural Characteristics of Surface Composite on Titanium Alloy Fabricated via a Novel Method of Friction-Stir Nitriding
}

\author{
Bo $\mathrm{Li}^{1, \mathrm{a}}$ \\ ${ }^{1}$ Shanghai Institute of Special Equipment Inspection and Technical Research, 399 North Nujiang \\ Road, Shanghai 200333, PR China \\ A libo@ssei.cn
}

Keywords: Friction-stir nitriding; Titanium alloy; Friction stir process; Microstructure; Surface Abstract. The microstructural evolutions and properties of the surface composite produced via the friction stir nitriding (FSN) method on Ti-6Al-4V alloy were investigated. Due to the optimized process parameters and suitable mixed gas proportion for a limited combustion reaction between $\mathrm{Ti}$ and $\mathrm{N}_{2}$, a composite layer with the thickness of $\sim 200 \mathrm{~m}$ beneath an outermost thin TiN layer was fabricated. The composite layer was composed of three microstructure types. Formation mechanisms for microstructure evolutions during FSN on titanium alloy were detailed in terms of three steps.

\section{Introduction}

Titanium alloys would have much wider usage in various industrial sectors if their wear-resistance could be significantly improved [1]. To solve this problem, the surface micro-structure of titanium can be modified via nitriding techniques, such as plasma, laser, and gas nitriding [2]. The nitrogen has a high solubility in -Ti to significantly strengthen the surface layer due to the formation of the compound layer containing of $\mathrm{TiN}$ and/or $\mathrm{Ti}_{2} \mathrm{~N}$ [2]. In the present paper, a novel nitriding method called friction-stir nitriding (FSN) is proposed. Multiple research results announced that friction stir processing (FSP) is emerging as a very attractive method used primarily to provide the localized modification of microstructures in the surface layers of metal components or to in-situ form a wear-resistant composite surface [3-5]. Actually, FSN is employed for titanium and its alloys depending on the basic principles of FSP technique and a limited combustion reaction of Ti in a mixed atmosphere of $\mathrm{Ar}_{2}$ and $\mathrm{N}_{2}$ gases during FSP procedure. It is well-known that the wearable $\operatorname{TiN}_{\mathrm{x}}$ compounds can be synthesized via a rapid combustion reaction between $\mathrm{Ti}$ and $\mathrm{N}_{2}$ gas [6]. Interests in this alternative nitriding method of FSN for preparing composite surface layer embedded in the Ti matrix are motivated by low cost production, the considerable surface-layer thickness and no debonding issues due to the inherent material continuity between the treated layer and substrate.

\section{Experimental Details}

$3 \mathrm{~mm}$ thick plates of Ti-6Al-4V alloy were used as parent material (PM) with duplex phases after annealing treatment. FSN procedure was performed using a professional FSP machine with a shoulder-pin tool of WC-13wt.\% Co matrix for the friction-stir behaviors. The tool was surrounded by a specialized gas-circulating system to continuously introduce a mixed atmosphere of $\mathrm{Ar}_{2}$ and $\mathrm{N}_{2}$ gases during FSN. The rate of $\mathrm{Ar}_{2}$ was maintained as $3 \mathrm{~L} / \mathrm{min}$, while that of $\mathrm{N}_{2}$ was only $0.3 \sim 0.6 \mathrm{~L} / \mathrm{min}$ to avoid the possible excess combustion of Ti. The basic process of FSN was similar to FSP procedure using the optimized parameters as follows: tool-shoulder plunge depth (D) $0.06 \mathrm{~mm}$, rotation speed (N) 375rpm, travel speed (V) $90 \mathrm{~mm} \cdot \mathrm{min}^{-1}$. Metallographic samples were observed using optical microscope (OM) and scanning electron microscope (SEM) system with an energy dispersive X-ray spectroscope (EDS) to determine the chemical compositions. The general phase identification was performed using an X-ray diffractometer (XRD) with $\mathrm{Cu} \mathrm{K}$ radiation at $40 \mathrm{kV}$ and $40 \mathrm{~mA}$ at $4 \% \mathrm{~min}$. Vickers hardness was measured at $0.1 \mathrm{~kg}$ load and $15 \mathrm{~s}$ indentation time. Ball-on-disk dry-sliding wear test was conducted using a $4.8 \mathrm{~mm} \mathrm{Si}_{3} \mathrm{~N}_{4}$ ball as at counter-face material room temperature. The rotation speed of friction unit, with a fixed radius of $3 \mathrm{~mm}$, was $430 \mathrm{rpm}$ for $25 \mathrm{~min}$ at a test load of $5.8 \mathrm{~N}$. 


\section{Results and Discussion}

Fig. 1a and its partial enlarged detail of Fig.1b give the typical microstructure of the outmost layer fabricated via FSN. The numerous connected grains with high density and in size of $2 \sim 3 \mathrm{~m}$ were present. The EDS result (Fig.1c) proved that the typical grains within the outermost thin layer were mainly TiN. The crown morphology was the inevitable product of FSP or FSN procedure [3]. Before an acceptable surface engineering application, the crown should be removed for flatness. After that, XRD was used to determine the constituent phases of the grinded surface composite by detecting several samples. By analyzing the 2 locations of three strong diffraction peaks after determining each phase, it was generally concluded that the main constituent phases were $\mathrm{TiN}_{2} \mathrm{Ti}_{2} \mathrm{~N}, \quad-\mathrm{Ti}, \quad-\mathrm{Ti}$ and a small amount of oxide. Fig.2a shows a cross-section appearance of the originally processed sample with the crown surface. As indicated in Fig.2b, the microstructure beneath the outmost thin layer was numerous and incompletely connected particles. The EDS result at Position(1) shows that the atomic number ratio of $\mathrm{Ti}$ to $\mathrm{N}$ is approximately $1.2: 1$. Fig.2c gives the unconnected particles distributed, embedded in the two-phase Ti alloy matrix at the transition-layer between the upper nitriding layer and the beneath stir zone (SZ). The EDS result at Position(2) shows that the proportions of $\mathrm{Ti}$ and $\mathrm{N}$ atoms are respectively $65.22 \%$ and $34.78 \%$. It implied that the particles in the transition-layer were mainly $\mathrm{Ti}_{2} \mathrm{~N}$. Fig.2d and Fig.2e are respectively the microstructures of SZ without nitrides and as-received PM. It indicated that the peak processing temperature exceeded the

-transus, resulting into that the prior -phase in PM experienced -transform behaviors. Ref.[7-9] pointed that on cooling after -transform during Friction stir welding (FSW) or FSP, -Ti can nucleate at triple-points to grain-boundary (GB), coarsen to cover the prior- GBs, and grow from the formed GB- into the neighboring prior- grains with Burgers orientation relation. Thus, the final microstructure of regions containing GB-
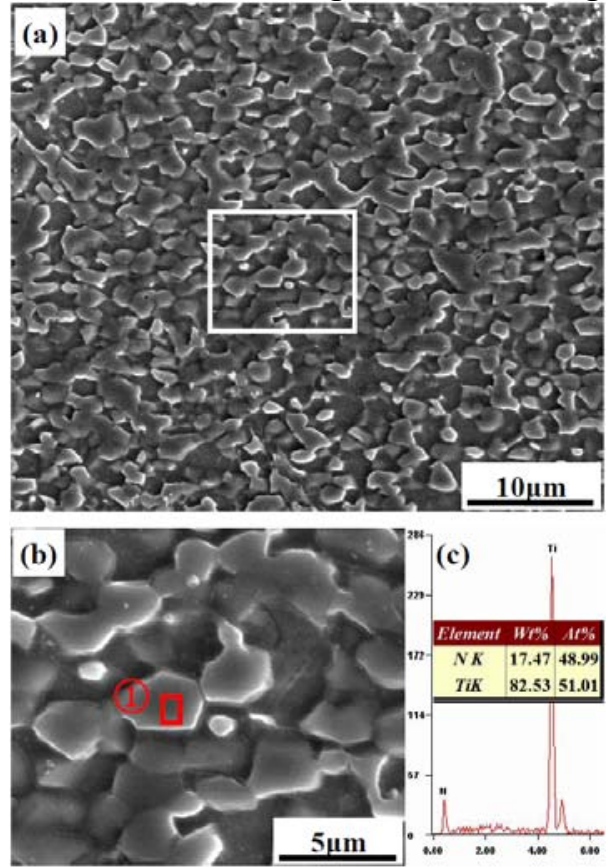

Fig.1 Microstructure of the outmost thin layer fabricated via FSN: a) typical morphology; b) partial enlarged detail; c) EDS result of Position(1). and acicular was formed (Fig.2d).


Fig.2 Vertical morphology of originally FSN sample:

a) macrostructure; b) microstructure beneath the outmost thin layer; c) microstructure at the transition-layer; d) microstructure of SN without nitrides; e) parent material.

The surface composite upper SZ had an approximate thickness of $200 \mathrm{~m}$ after removing the crown (Fig.3a). Fig.3b gives the morphology of the transition-layer exhibiting three typical layers numbered ' 1 ', ' 2 ' and ' 3 ' in Fig.3b. A gradient feature for vertical distribution or rank of nitride-particles embedded in the matrix was present in the transition-layer. Furthermore, the EDS result (Fig.3c) at Position(1) in Fig.3b implied that the SZ regions in Layer-3 lacked nitrides but contained $\operatorname{Ti}(\mathrm{N})$ solid solutions. Fig.3d gives the microstructure of a position in the transition-layer. The elongated prior- regions with acicular -Ti were present. Along the GBs among the adjacent 
regions, particles were precipitated from the GB- . The EDS result (Fig.4e) at Position(2) implied that the precipitated particles were nitrides of $\mathrm{Ti}_{2} \mathrm{~N}$. Thus, it was believed that part of nitride-particles were generated according the dissolution and precipitation mechanisms: $\quad-\mathrm{Ti} \rightarrow \quad-\mathrm{Ti}(\mathrm{N}) \rightarrow \mathrm{Ti}_{2} \mathrm{~N}$.

Fig. 4a shows the microstructure characteristics by a horizontal top-view on the surface composite after removing the crown and the outmost thin layer. The EDS results from Fig.4b (Position(1)) and Fig.4c (Position(2)) implied that the bulky material regions and the particles surrounding them were titanium nitride, which is a typical material with a wide range of stoichiometry $\left(\operatorname{TiN}_{1-\mathrm{x}}, 0<\mathrm{x}<0.61\right)$ [3]. The composite was composed of three types of typical microstructures, which are marked in Fig.4a and depicted as follows: (i) Type-A was bulky material containing nitrides of titanium nitrides with excess $\mathrm{Ti}$ by combustion reaction procedure; (ii) Type-B was dense nitride-particles mainly of titanium nitrides embedded in the matrix; (iii) Type- $\mathrm{C}$ was poor-nitride narrow bands, as arrowed in Fig.4a, with a small number of precipitated nitride particles.

Vickers hardness testing results indicated that the average micro-hardness of the composite surface was $912 \mathrm{Hv}_{0.1}$, while the highest value was $1103 \mathrm{Hv}_{0.1}$. The COF variations of the composite surface without the crown were given as Fig.4d. The average value of COF was 0.33, which was obviously lower than that of Ti-6Al-4V PM as 0.54 under the same testing conditions. Although the microstructure of composite layer fabricated via FSP was not uniform in the micro-scale structure, the wear-resistance was not evidently suppressed due to the high density of wearable nitrides. Therefore, the COF curve exhibits a relatively steady-state behavior with small fluctuations (Fig.4d).
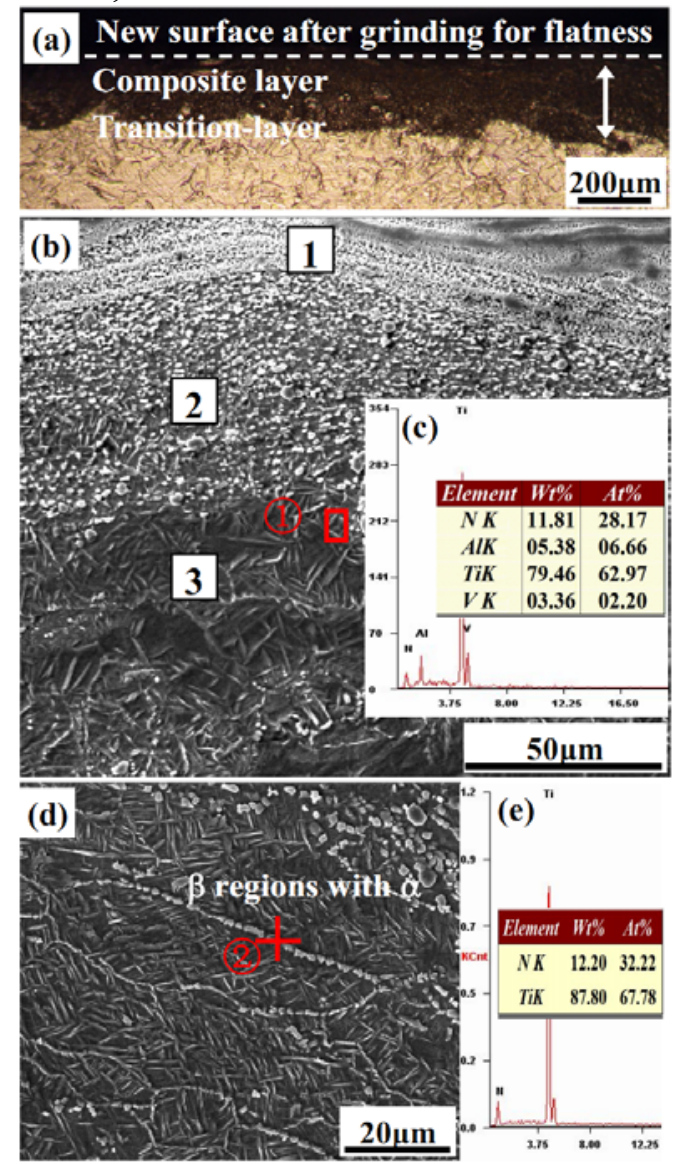

Fig.3 Vertical morphology of the transition-layer: a) macrostructure without the crown surface; b) microstructure of three typical layers; c) EDS of Position(1); d) microstructure of the nitride precipitates; e) EDS of Position(2).
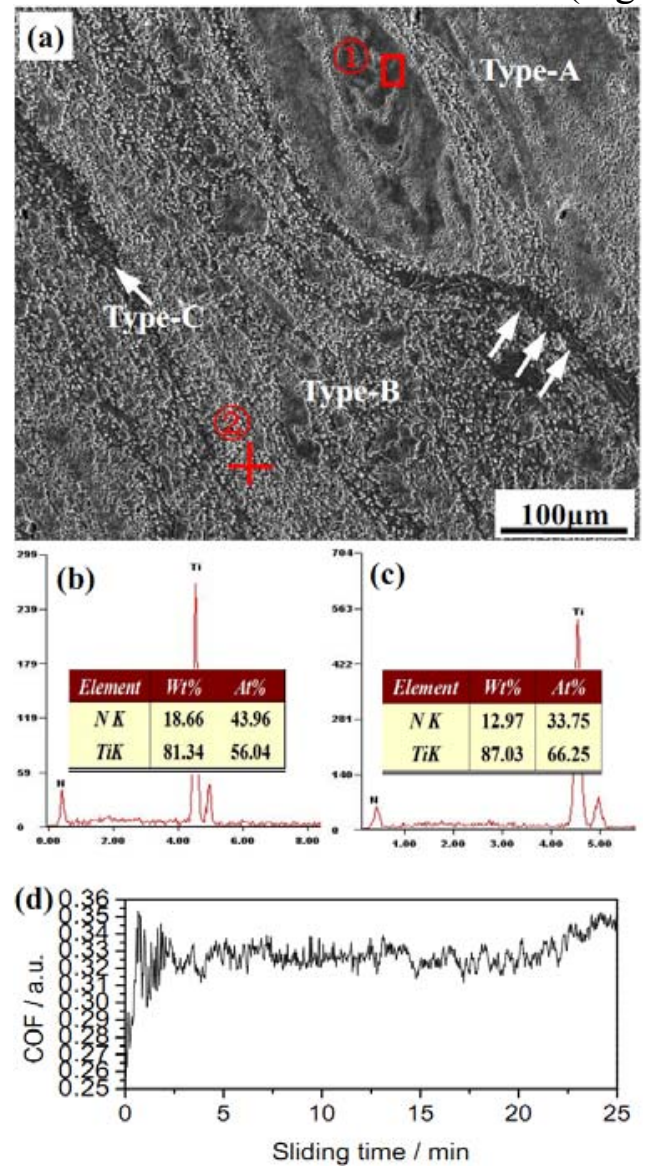

Fig.4 Microstructure of the composite surface on the top view: a) three typical microstructures; b) EDS of Position(1); c) EDS of Position(2); d) COF curves of the grinded surface.

During FSN procedure, the microstructure evolutions involved multiple formation mechanisms, which were experimentally proved in this work and detailed in terms of three steps. 


\section{Step-I (friction heating and combustion reaction)}

Heats were generated and severely concentrated beneath the rotating tool-shoulder by continuously friction, resulting the extremely rise in temperature. Then the combustion reaction between Ti and $\mathrm{N}_{2}$ occurred [10]. Due to the high-speed tool-stirring behavior, the flow of plasticized material beneath the tool-shoulder constantly provided fresh Ti to react with $\mathrm{N}_{2}$. Thus, the acceptable percent conversion of titanium nitrides could be ensured. On the other side, the optimized process parameters and suitable $\mathrm{N}_{2}$ proportion in the mixed atmosphere contributed to shorten the heating period, limit the combustion degree and avoid the large-scale spread of possibly violent $\mathrm{Ti}$-fire.

Step-II (nitride melting, dispersion by stir and cold-curing)

The maximum temperature for the reaction system of Ti and $\mathrm{N}_{2}$ was more than the melting points of $\mathrm{Ti}$ and TiN [10]. Thus, the products would be the liquid state. Within the flow filed of severely plasticized materials by stirring beneath the tool-shoulder and around the pin, the liquid materials with low flow stress could be dispersed and migrated. That could effectively ensure to obtain a considerable thickness of the composite layer. Step I and II would be finished after that the rotating, traveling tool left away from the processed zone behind it. As the cold-curing procedure, the bulky nitride materials with different sizes and shapes were formed in the composite layer.

\section{Step-III ([N]-dissolution and precipitation of nitride)}

The melted composites produced during FSP could provide considerable activated $[\mathrm{N}]$. The $[\mathrm{N}]$ could be dissolved into the surrounded melting Ti and diffused into the adjacent solid Ti alloy. During the cooling period, numerous particles of titanium nitride particles were precipitated from the Ti(N) solid solutions, especially the $-\operatorname{Ti}(\mathrm{N})$ which had a higher nitrogen solubility than $-\operatorname{Ti}(\mathrm{N})$.

\section{Summary}

Depending on the optimized process parameters and suitable $\mathrm{N}_{2}$ gas proportion, a composite layer on the Ti-6Al-4V alloy with a considerable thickness of more than 200 m beneath an outermost thin layer of TiN were fabricated via FSN in a mixed $\mathrm{Ar}_{2}$ and $\mathrm{N}_{2}$ atmosphere. After removing the crown, the composite layer, with a high average hardness value and lower COF, was composed of bulky nitrides and densely distributed nitride-particles embedded in the Ti-matrix. Effective but limited combustion reaction between $\mathrm{Ti}$ and $\mathrm{N}_{2}$ was the main formation mechanism for titanium nitrides.

\section{Acknowledgements}

The work was supported by China Postdoctoral Science Foundation funded project (2015M580342) and the National Natural Science Foundation of China (Grant No.51505293).

\section{References}

[1] Y.S. Tian: Mater. Lett. Vol.64(2010), p.2483.

[2] A. Zhecheva, W. Sha, S. Malinov and A. Long: Surf. Coat. Tech. Vol.200(2005), p.2192.

[3] Z.Y. Ma: Metal. Mater. Trans. A. Vol.39(2008), p.642.

[4] Q. Zhang, B.L. Xiao, Q.Z. Wang and Z.Y. Ma: Mater. Lett. Vol.65(2011), p.2070.

[5] F.Y. Tsai and P.W. Kao: Mater. Lett. Vol.80(2012), p.40.

[6] R. Marin-Ayral, C. Pascal, F. Martinez and J. Tedenac: J. Eur. Ceram. Soc. Vol.20(2000), p.2679.

[7] S. Mironov, Y. Zhang, Y.S. Sato and H. Kokawa: Scripta. Mater. Vol.59(2008), p.27.

[8] H.J. Liu, L. Zhou and Liu Q: Scripta. Mater. Vol.61(2009), p.1008.

[9] A. Pilchak and J. Williams: Metal. Mater. Trans. A. Vol.42(2011), p.773.

[10] H. Kudo and O. Odawara. J. Mater. Sci. Vol.24(1989), p.4030. 